\title{
Hunting for the lowest-mass exoplanets
}

\author{
Francesco Pepe ${ }^{1}$, Michel Mayor ${ }^{1}$, Christophe Lovis ${ }^{1}$, Willy Benz ${ }^{2}$, \\ François Bouchy ${ }^{3}$, Xavier Dumusque ${ }^{1}$, Didier Queloz ${ }^{1}$, Nuno C. \\ Santos $^{4}$, Damien Ségransan ${ }^{1}$ and Stéphane Udry ${ }^{1}$ \\ ${ }^{1}$ Observatoire Astronomique de l'Université de Genève, 51, ch. des Maillettes, CH-1290 \\ Versoix, Switzerland, email: Francesco.Pepe@unige.ch \\ ${ }^{2}$ Physikalisches Institut der Universität Bern, Sidlerstrasse 5, CH-3012 Bern, Switzerland \\ ${ }^{3}$ Institut d'Astrophysique de Paris, 98bis, bd Arago, F-75014 Paris, France \\ ${ }^{4}$ Centro de Astrofísica da Universidade do Porto (CAUP), Rua das Estrelas, P-4150-762 Porto, \\ Portugal
}

\begin{abstract}
In order to understand general planet characteristics and constrain formation models it is necessary to scan over the widest possible parameter range of discovered systems. Due to detection biases, the domain of very-low mass planets had remained poorly explored. Only with improving measurement precision it has been possible to enter in the sub-Neptune mass range. The HARPS planet search program has been particularly efficient in detecting such ice giants and super earths. The present talk will summarize the obtained results and the characteristics of the low-mass population of exoplanets.
\end{abstract}

Keywords. techniques: radial velocities, planetary systems

\section{The discovery of a rich population of low-mass planets on tight orbits}

Today, more than 500 extrasolar planets have been discovered. Most of the detected exoplanets have been found by using precise measurements of stellar radial velocities. The planetary mass estimate from Doppler measurements is directly proportional to the amplitude of the stellar reflex motion. Our progress to detect very-low-mass planets are directly related to the progress done to improve the sensitivity and stability of spectrographs. In 1989, the detection of HD $114762 \mathrm{~b}$, a companion of 11 Jupiter masses to a metal deficient F- star was obtained with spectrographs allowing Doppler measurements with a precision of some $300 \mathrm{~m} / \mathrm{s}$ (Latham et al. 1989). Fifteen years ago, the precision achieved by any team searching for exoplanets was of the order of $15 \mathrm{~m} / \mathrm{s}$. Today, the instrumental precision achieved with the HARPS spectrograph at La Silla Observatory is better than $0.5 \mathrm{~m} / \mathrm{s}$ (Mayor et al. 2003). At this level of precision we are mostly limited by the intrinsic variability of stellar velocities induced by diverse phenomena (acoustic modes, granulation, magnetic activity). However, by adopting an improved observing strategy, we have already some indications that planetary signals as small as a tiny fraction of a meter per second are detectable.

This progress in instrumentation and observing strategy have made possible the discovery of a rich population of super-Earths and Neptune-mass planets in tight orbits around solar-type stars (Mayor \& Udry 2008).

The name "super-Earth" is used to qualify planets more massive than the Earth but with masses smaller than 10 Earth masses, a category of planets absent in the solar system. We mention here a few landmark discoveries of these low-mass planets orbiting solar-type stars. Limiting ourself to planets in the super-Earth range we can mention: $\mu$ Ara c with a mass of $10.5 M_{\oplus}$ and a period of 9.7 days (Santos et al. 2004, revised in 


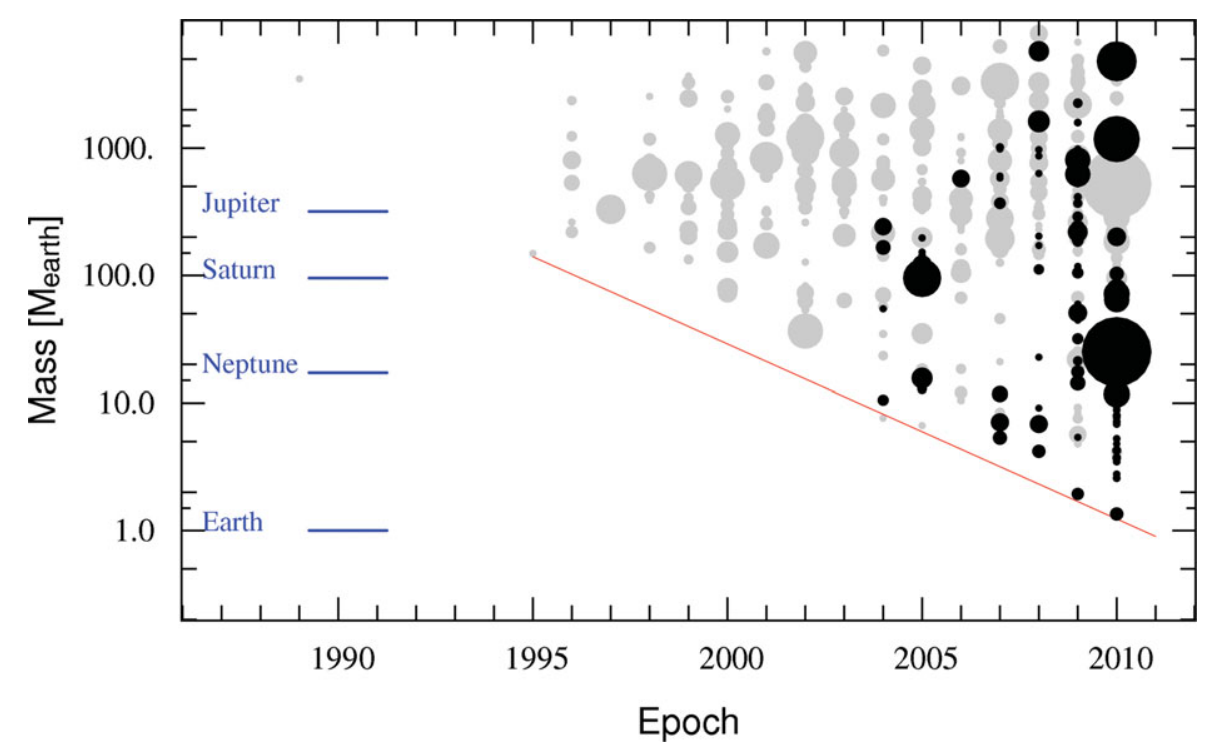

Figure 1. Minimum mass of planets detected by Doppler spectroscopy as a function of epoch of discovery. This figure illustrates the impressive progress made in detection sensitivity over the past 15 years. Black symbols indicate HARPS discoveries. Dot size is related to orbital semi-major axis.

Pepe et al. 2007), HD $69830 \mathrm{~b}$ with a mass of $10.2 M_{\oplus}$ and a period of 8.7 days (Lovis et al. 2006), HD $40307 \mathrm{~b}$, c, d, a system with three super-Earths with masses comprised between 4 and $9 M_{\oplus}$ and periods from 4 to 20 days (Mayor et al. 2009b). We also have to mention the exceptional system around HD 10180, with 7 planets of which one with a mass as small as $1.4 M_{\oplus}$ on a tight orbit with a period of 1.17 day (Lovis et al. 2011). In addition to these early detections of super-Earths orbiting solar-type stars, we also have to mention the discoveries of super-Earths hosted by M-dwarfs: GJ $876 \mathrm{~d}$, a planet with a mass of $5.9 M_{\oplus}$ and a period of 1.94 day (Rivera et al. 2005; Correia et al. 2010), GJ 581 c, d, e with masses of 5, 7 and $1.9 M_{\oplus}$ (Udry et al. 2007; Mayor et al. 2009a). It is impressive to see that all these super-Earths are part of rich multi-planetary systems with 3 to 7 planets per system. The remarkable progress of instrumentation in the last 15 years is obvious in Fig. 1. The masses of planetary companions are plotted as a function of the epoch of their discovery. The mass of HD $10180 \mathrm{~b}$ (Lovis et al. 2011) is a factor 100 smaller than the mass of 51 Peg b (Mayor \& Queloz 1995).

\section{Reaching sub-meter-per-second precision}

There is no doubt that successful detections depend on the observational precision, in particular when searching for signals with semi-amplitudes below $1-2 \mathrm{~m} \mathrm{~s}^{-1}$, as it is the case for the presented program. The instrument used for our program, ESO's HARPS, is recognized as the most precise RV instrument worldwide. Five years of observations have proved that, on quiet and bright dwarf stars, a radial-velocity precision well below $1 \mathrm{~m} \mathrm{~s}^{-1}$ can be achieved on both short-term (night) and long-term (years) timescales. A direct confirmation is offered by the raw RV dispersions measured for the candidates discovered with HARPS. Furthermore, when binning the data-points over several days to remove meso-granulation effects of the stellar surface, the radial-velocity dispersion 
can be reduced below $30 \mathrm{cms}$ over over observational period of several years (see e.g. Lovis et al. (2006) describing the detection of three Neptunes orbiting HD 69830).

The recent impressive HARPS discoveries made us aware of the fact that discovering Earth analogs is already within the reach of HARPS, although important questions still remain open: How frequent are low-mass planets? At what level is the detection bias? Where is the precision limit set by stellar noise? Can we detect low-mass planets in the habitable zone of their parent star if sufficient observations on a high-precision instrument are invested? Driven by the very encouraging results of the HARPS High-Precision Program (Udry \& Mayor 2008) and the hints for high planet occurrence announced by Lovis et al. (2009), we have decided to investigate these questions in further detail. For this purpose, we have defined a specific program on HARPS for the search of rocky planets in the habitable zone HZ based on Guarantee Time Observations (GTO) awarded to us by ESO for the upgrade and maintenance of the HARPS spectrograph. In this program we have focused on 10 quiet, non-rotating, non-active stars of the original HARPS high-precision program and the observational strategy was optimized. The program is being carried out presently and the first results are being obtained.

In order to illustrate the kind of objects we are dealing with and the level of precision which can be attained with HARPS, we shall focus a moment on HD 10700, also known as Tau Ceti. This is the most quiet star in our sample and shows a dispersion of only $0.92 \mathrm{~m} \mathrm{~s}^{-1}$, despite the large number of data points spanning 6 years of observations. What is even more remarkable it that none of the parameters shown in Fig. 2, i.e. the radial velocity, the chromospheric activity indicator $\log \left(R_{\mathrm{HK}}^{\prime}\right)$, and the average line bisector of the cross-correlation function $B I S$, show any trend over the 6 years of observations. Most amazing is the line bisector, which has a dispersion of about $0.5 \mathrm{~m} \mathrm{~s}^{-1}$, confirming the extreme stability of HARPS' instrumental profile (IP).

One may also wonder how strongly stellar activity may influence the radial-velocities. We refer to Dumusque et al. (2011a) and references therein for a detailed discussion. In the specific case of Tau Ceti we observe only little RV jitter despite the fact that the $\log \left(R_{\mathrm{HK}}^{\prime}\right)$ seems to vary significantly at some periods, while it remains stable over other periods. As an example, Fig. 3 shows the radial velocity and the $\log \left(R_{\mathrm{HK}}^{\prime}\right)$ over a single seasons. Significant variations (in terms of photon noise) of the $\log \left(R_{\mathrm{HK}}^{\prime}\right)$ can bee seen, while no correlated variation is detected in the radial velocity. This is better illustrated in Fig. 4 where the correlation between the two quantities is plotted. We deduce that in the specific case of Tau Ceti, the radial velocity is marginally affected by stellar activity. This behavior is expected for late G and K dwarfs (Dumusque et al. 2011a).

\section{The case of Gl 581}

Recently, Vogt et al. (2010) have announced the discovery of a 5th and 6th planet around the star Gl581. This detection resulted from the combination of HARPS and HIRES data. When fitting a given model to the HIRES and HARPS data equally weighted, the residuals of the HARPS data are of the order of $1 \mathrm{~m} \mathrm{~s}^{-1}$, while the for the HIRES data they are significantly higher than $2 \mathrm{~m} \mathrm{~s}^{-1}$. From this fact we concluded that the HIRES data where not sufficiently precise to allow the detection of a planet with $\mathrm{m} \mathrm{s}^{-1}$ amplitude. On the other hand, the high number of HARPS data and the good time sampling, would allow to see a potential 5th and 6th signal with HARPS data only. Therefore, we have investigated the presence of possible related signals in the HARPS data only. Fig. 5 shows the generalized Lomb-Scargle periodogram (GLS) of the residuals after fitting the four already know planets. It appear evident, that none of the residual peaks is significant, being all of them well below the $10 \%$ false-alarm probability (FAP) 

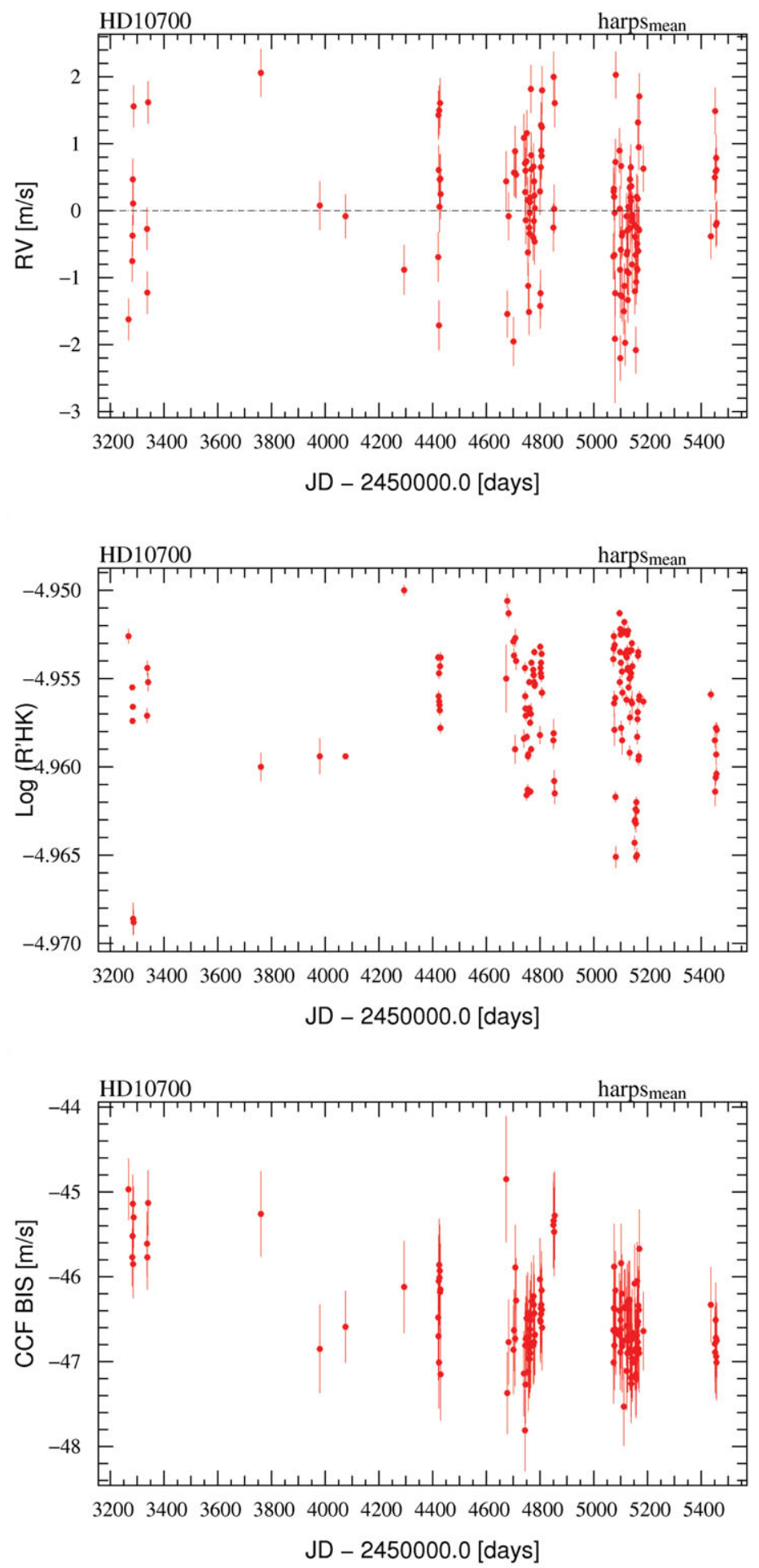

Figure 2. Radial velocities, chromospheric activity indicator $\log \left(R_{\mathrm{HK}}^{\prime}\right)$, and line bisector $C C F B I S$ of HD 10700 versus time. 

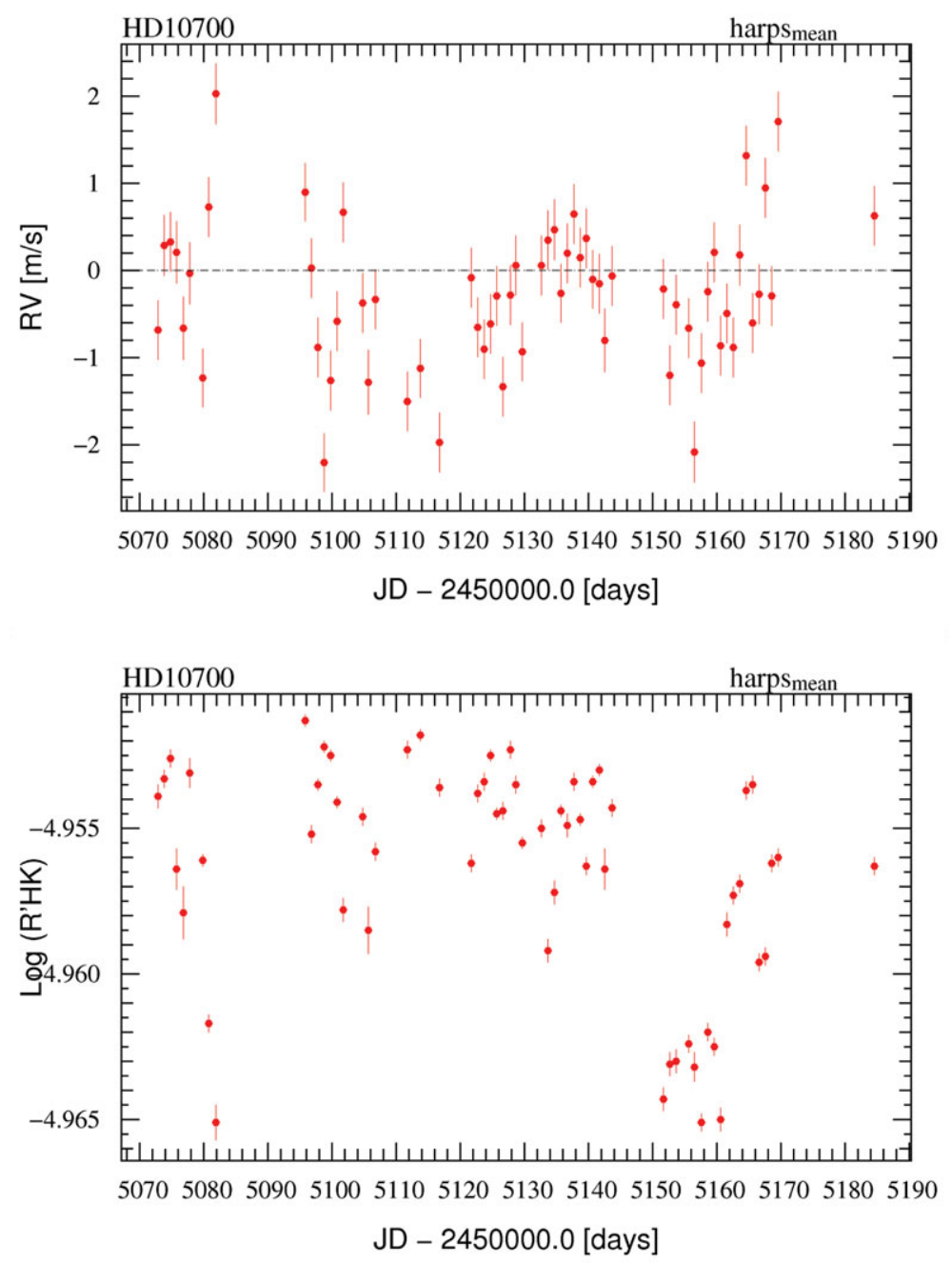

Figure 3. Radial velocitiy and chromospheric activity indicator $\log \left(R_{\mathrm{HK}}^{\prime}\right)$ in a single season.

threshold. It is however true that, if the eccentricity of the planet at $P=66$ days is fixed at 0 , then a peak at $P=33$ days appears. However, the resulting 'signal' has a FAP of several percent, and in particular, its period is significantly different from the period of 37 days of the f-planet announced by Vogt et al. The same discussion applies for the g-planet. We conclude from this that the more precise HARPS data show no evidence for the existence of potential 5th and 6th planets around Gl 581 with orbital parameters as announced by Vogt et al. We would like to refer at this point to two independent studies carried out by Gregory (2011) and Andrae et al. (2010) and published shortly after the IAUS 276 Symposium. They proof that the signals detected by Vogt et al. are statistically not relevant. Both studies indicate that, using the same data sample on which Vogt et al. based their analysis, the most likely model is the one with 4 planets. Furthermore they show that HIRES data only would degrade the quality of the found solutions. 


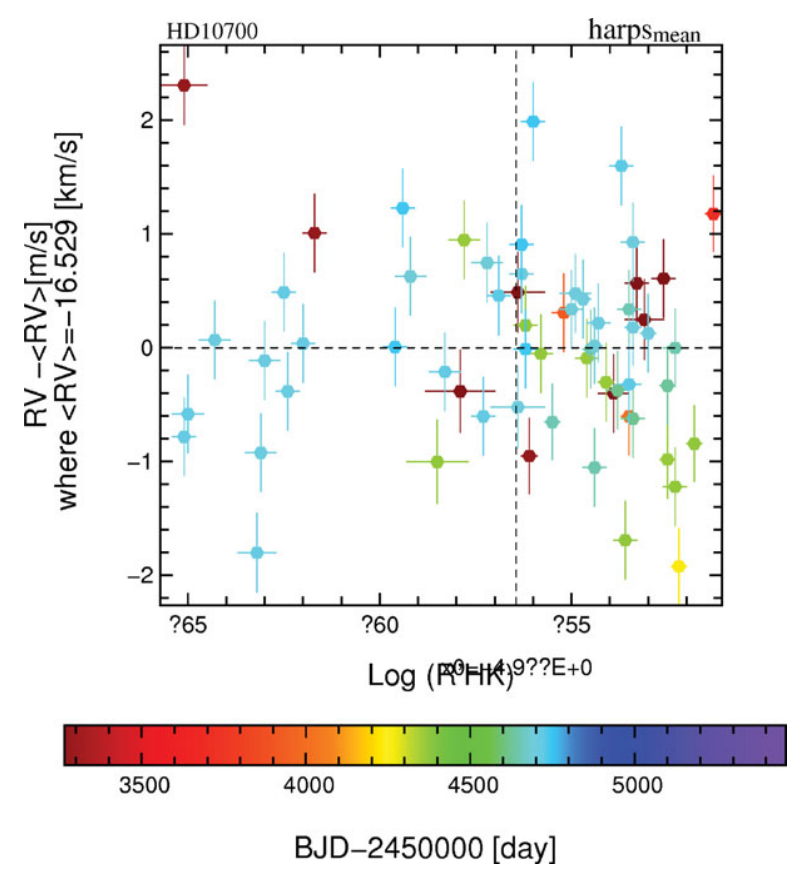

Figure 4. Correlation plot of radial velocity versus chromospheric activity indicator $\log \left(R_{\mathrm{HK}}^{\prime}\right)$.

\section{The HARPS program to search for very low mass planets}

HARPS is a vacuum-operated high-resolution spectrograph $(\mathrm{R}=115,000)$, fiber-fed, optimized to provide stellar radial-velocity measurements with extreme precision (Mayor et al. 2003). As a reward for its construction, the HARPS consortium has received guaranteed observing time (GTO) to carry out an extrasolar planet search in the southern hemisphere (500 observing nights over 5 years). More than $60 \%$ of the total HARPS GTO observing time has been devoted to two sub-programs having the aim of detecting very low-mass planets. The first of these sub-programs comprises some 400 stars which are non-active, slow rotators, not in spectroscopic binary systems, and were selected from the large volume-limited sample measured for several years with the CORALIE spectrograph on the $1.2 \mathrm{~m}$-Euler telescope at la Silla Observatory. The second sub-program consists of a volume-limited sample of about $120 \mathrm{M}$ dwarfs at the bottom of the main sequence, also selected to be slow rotators and not members of spectroscopic binary systems.

What are the limits presently achieved in terms of radial velocity precision? Several sources of noise can be identified:

- As a result of the efficiency of the cross-correlation technique, a photon noise level of only a fraction of a meter per second is achieved in a few minutes for most of our targets. Sometimes the exposure time is shorter than the typical periods of stellar acoustic modes. In a few minutes, the full amplitude of the stellar velocity variations resulting from acoustic modes could be as large as several meters per second. Long integrations compared to acoustic mode periods are sufficient to have acoustic noise residuals smaller than $0.2 \mathrm{~m} / \mathrm{s}$ (rms). For most stars, integrations of 15 minutes are sufficient.

- Dumusque et al. (2011a) have shown that stellar granulation in solar-type stars can induce radial velocity variability comparable to or larger than $1 \mathrm{~m} / \mathrm{s}$ on longer timescales compared to acoustic modes. Several measurements spanning several hours are requested to damp the granulation noise. 


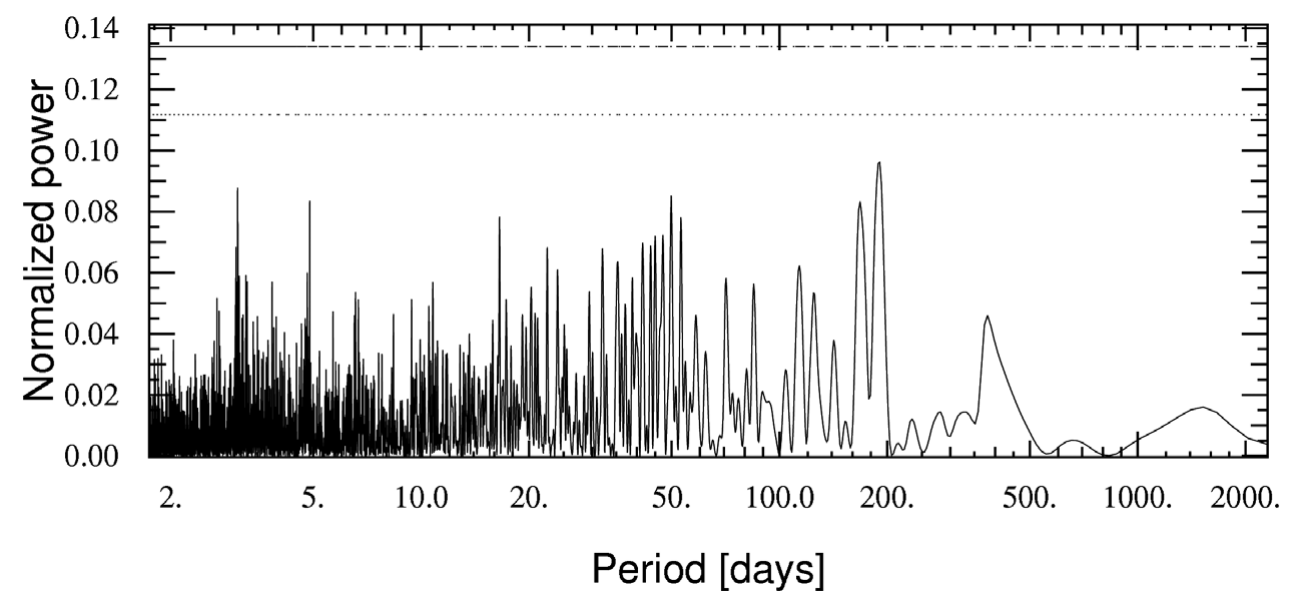

Figure 5. Generalized Lomb-Scargle periodogram of the HARPS radial-velocities of G1 581. The horizontal line mark the $10 \%$, respectively $1 \%$ FAP limits.

- Any anisotropies in stellar atmospheres related to magnetic activity will induce radial velocity variations at the stellar rotation period. The amplitude of the radialvelocity jitter is related with stellar chromospheric activity. If we want to search for very low mass planets we need to carefully select "non-active" stars. The reemission in the core of the calcium $\mathrm{H}$ and $\mathrm{K}$ lines is a good indicator of the chromospheric activity and has been used for the selection of the stellar sample.

- The analysis of the radial velocity variations of several solar-type stars has recently revealed well-defined variations of several $\mathrm{m} / \mathrm{s}$ on rather long periods (more than five years). These velocity variations are strongly correlated with the mean shape of absorption lines and chromosperic indicators like $\mathrm{Ca}$ II H \& $\mathrm{K}$ core emission. These variations are related to the stellar analogs of the solar magnetic cycle. This effect has been observed in stars with rather modest chromospheric activity levels (e.g. $\log R_{\mathrm{HK}}^{\prime}$ around -4.90 , see Lovis et al. 2011, in prep.). Any long-term drift in stellar radial velocities cannot be a priori attributed to long period planets if a careful check of the long-term behavior of the line bisector and other activity indicators has not been performed.

- Finally, we still have instrumental noise. Lovis \& Pepe (2007) have considerably improved the precision of the wavelength of thorium lines as well as the number of lines to be used for the calibration of the spectrograph. Pressure changes in the plasma with the aging of the ThAr calibration lamp induce a very small shift in the wavelengths. As this effect is smaller for thorium lines than argon, we can use this differential effect to correct the aging effect. Long term drifts have thus been reduced below $0.3 \mathrm{~m} / \mathrm{s}$ over timescales of several years. The scrambling effect in optical fibers is excellent... but not perfect and some sub-meter per second error could result from imperfect guiding.

The global budget of all these errors is difficult to determine. The best estimation of the lower limit of the quadratic sum of the different components of the noise is provided by the residuals observed around fitted radial velocity curves. Several stars with a very large number of velocity measurements spanning several years have residuals with a dispersion as low as $0.6 \mathrm{~m} / \mathrm{s}$ (when binning the data over a few days). For stars with larger chromospheric activity, we can obviously have larger residuals.

This is the precision presently achieved for the HARPS program, for which we have derived preliminary results for the population of low mass planets, as discussed in the next section. If we are searching for low-mass planets on rather long periods, it could 


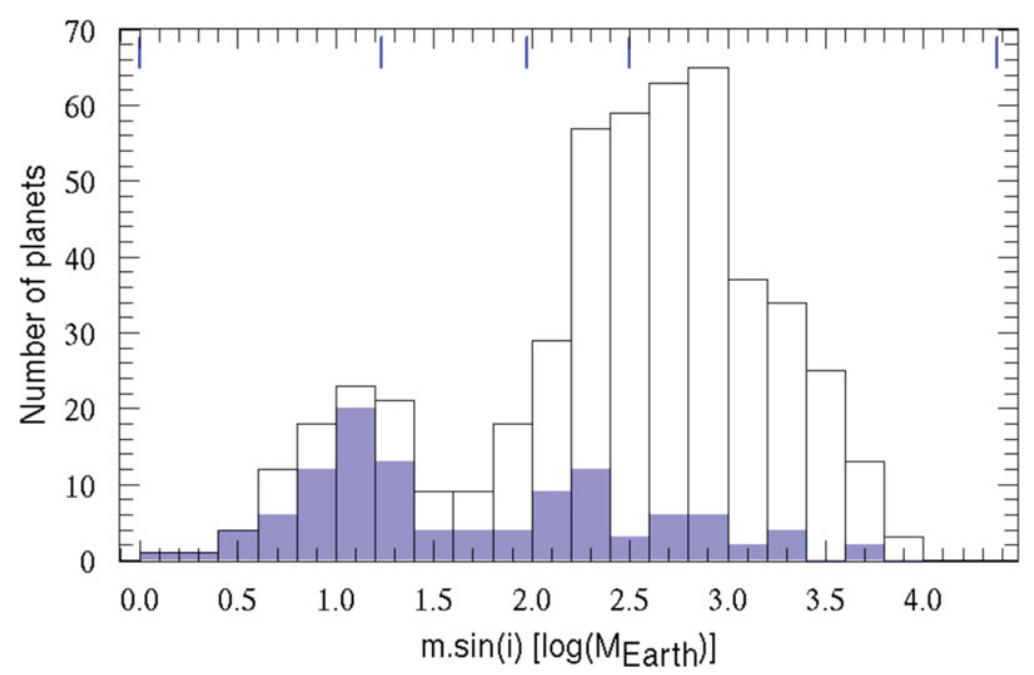

Figure 6. Mass distribution of all detected planets. The contribution of the HARPS program (solid histogram) for the detection of very low mass planets is evident.

be useful to bin the measurements done on $\mathrm{N}$ consecutive nights. This procedure could help to damp the noise induced by chromospheric activity, with a time scale comparable to the stellar rotation period. First experiments done on stars with a large number of measurements have shown that the residuals decrease to $0.3-0.5 \mathrm{~m} / \mathrm{s}$ after binning over ten consecutive nights.

\section{Emerging characteristics of low-mass planets and their host star}

We are still far from having a detailed and unbiased view of the population of planets with masses in the range of super-Earths and Neptunes. Nevertheless, we can already notice a few emerging properties. The study of planet hosts themselves also provide additional information to constrain planet formation. In particular the metallicity of the parent stars seems to be of prime importance for models of planetary formation.

\subsection{The mass distribution}

The mass distribution of all detected planets is illustrated in Fig. 6. In this plot the contribution of the HARPS program for the detection of very low mass planets is evident. Due to the better detection sensitivity of Doppler spectroscopy for massive and/or short period planets, we still have a strong bias against the detection of low-mass planets, especially if they are on long-period orbits.

The bimodal aspect of the mass distribution is a clear indication that the decrease of the distribution for masses less than about one mass of Jupiter is not the result of a detection bias, but is real. The extrapolation by a power-law distribution, as for example $f(m) \sim m^{-1}$, to estimate the number of planets with a mass smaller than the mass of Jupiter is certainly not justified. The observed bimodal shape of the mass distribution from gaseous giant planets to the super-Earth regime provides an interesting constraint for planetary formation scenarios. The planetary formation simulations carried out by Mordasini et al. (2009a,b) also predict a bimodal distribution for that range of planetary mass. In addition these simulations also predict a sharp rise in the mass distribution at a few Earth masses and below. This domain of mass is still at the limit of present instrumental sensitivity. Nevertheless, once again the expected shape of this theoretical 


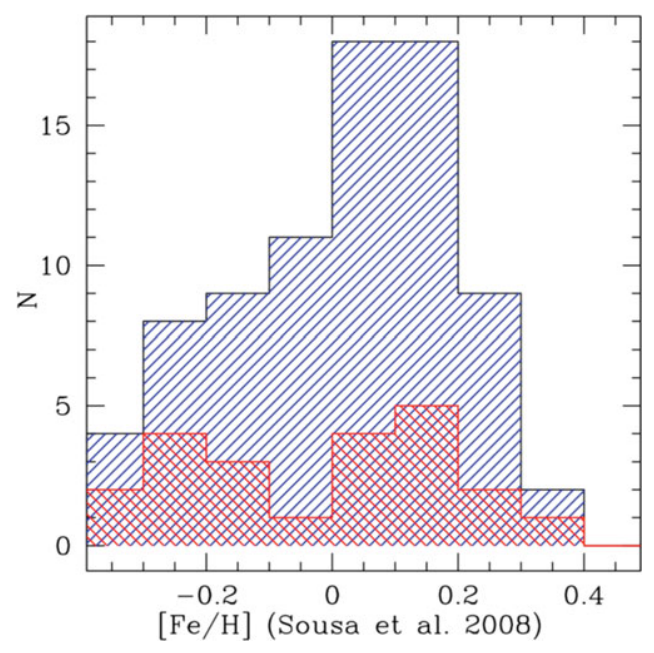

Figure 7. Correlation of the frequency of planets as a function of host star metallicity. Planetary systems with masses smaller than $20 M_{\oplus}$ are frequently hosted by metal-deficient stars (cross-hatched histogram), at the opposite of the correlation observed for gaseous giant planets (hatched histogram).

mass distribution from 10 down to $1 M_{\oplus}$ is clearly not an exponential and any estimate of the frequency of Earth-twins based on an exponential extrapolation is completely unjustified.

\subsection{The frequency of low-mass multiplanet systems}

With the HARPS data presently available from the high-precision sample, we have 48 stars with well-characterized planetary systems. More than $50 \%$ of these systems are multiplanetary. Four of them have 4 planets and the amazing system HD 10180 is the host of 7 planets (Lovis et al. 2011), one of them having a mass as small as $1.5 M_{\oplus}$.

\subsection{The correlation with the metallicity of host stars}

The correlation between the occurrence of gaseous giant planets and the metallicity of host stars is striking. Based on large planetary surveys this correlation is well established by independent teams (Santos, Israelian \& Mayor 2001, 2004; Fischer \& Valenti 2005). We have a completely different result if we examine the metallicity of host stars for systems having all planets less massive than $40 M_{\oplus}$. We do not have any correlation between the presence of these low-mass planets and the host star metallicity (see Fig. 7), a result already mentioned by Udry et al. (2006) and Sousa et al. (2008), based at that time on a very limited number of stars. With the present study, this lack of correlation with the host star metallicity is robust. The mean metallicity of the 28 planetary systems with planets less massive than $40 M_{\oplus}$ is $[\mathrm{Fe} / \mathrm{H}]=-0.12$, a metallicity not so different from the mean metallicity of stars in the solar neighborhood.

\subsection{The occurrence of low-mass planets orbiting solar-type stars}

The occurence of low-mass planets on tight orbits has been estimated by Lovis et al. (2009). For planets with masses between $\sim 5$ and $50 M_{\oplus}$ and periods shorter than 100 days, we have detected low-mass planets orbiting about $30 \%$ of the stars in the HARPS sample. A more complete estimate is currently in progress, based on the present, more complete survey. 


\subsection{Searching for Earth-type planets in the habitable zone}

The programme devoted to the study of the population of super-Earths and Neptune-type planets is still continuing at la Silla for four additional years after the end of the GTO time. In addition, a new exploratory program has been initiated with the goal of pushing the HARPS precision a little further and try to detect super-Earths in the habitable zone of very nearby $\mathrm{G}$ and $\mathrm{K}$ dwarfs. An adequate strategy to damp the acoustic and granulation noise sources has been implemented. The sample is limited to only 10 bright non-active stars. Already, low-mass planets have been detected on three stars members of that small sample, see Pepe et al. (2011). The radial velocity signal for one of these planets is as small as $\mathrm{K}=0.6 \mathrm{~m} / \mathrm{s}$. Furthermore, simulations done by Dumusque et al. (2011b) have demonstrated the possibility with the HARPS spectrograph, the present observing strategy and precision, to detect a $2.5-M_{\oplus}$ planet orbiting a non-active K-dwarf in its habitable zone (see Fig. 8).

Some technical improvements are still feasible to increase the sensitivity and stability of cross-correlation spectrographs like HARPS. A better scrambling of the input beam could be achieved by new optical fibers with octogonal cross sections. These new fibers will strongly diminish the already very small effect of input conditions (guiding errors, variable seeing and focus) on the spectrograph illumination, a mandatory condition to achieve $0.1 \mathrm{~m} / \mathrm{s}$ precision. To secure the stability of radial velocity measurements over a span of several years at the level of $0.1 \mathrm{~m} / \mathrm{s}$ or better, we must have a calibration device better than the existing ThAr lamps. Developments of laser frequency combs adapted to the resolution and wavelength coverage of HARPS will provide the requested stability (Wilken et al. 2010).

A photon noise on the Doppler signal at the level of $0.1 \mathrm{~m} / \mathrm{s}$ requires a rather large telescope size to achieve the needed signal-to-noise ratio in a reasonable exposure time. The ESPRESSO project, presently in development, to be implemented on the 8.2-m VLT telescope at Paranal is designed to achieve the $0.1 \mathrm{~m} / \mathrm{s}$ Doppler precision and stability on the long term (Pepe et al. 2010). The ESPRESSO project can also be seen as a precursor for an even more ambitious stable spectrograph, the CODEX project presently at the study phase level for the 42-m E-ELT telescope, to be implemented by ESO at Cerro Armazones (Chile) in the next decade (Pasquini et al. 2010).

We have to keep in mind that for stars with the lowest chromospheric activity, we still do not know the true level of radial velocity jitter. Analysis of the radial velocity scatter of HARPS measurements for non-active stars suggest a minimum jitter of $0.5 \mathrm{~m} / \mathrm{s}$ or less. This stellar variability, depending on the changing number and phase of magnetic spots (or other features) will be difficult to model. Preliminary studies show that non-active $\mathrm{K}$ dwarfs will be the most suitable targets to search for Earth twins. A large number of Doppler measurements has the potential to overcome the effects of the stellar intrinsic variability and permit detections of planetary signals of $0.2 \mathrm{~m} / \mathrm{s}$ or less.

The discovery of radial velocity variations associated with solar cycle analogues with full amplitude as large as $10 \mathrm{~m} / \mathrm{s}$ seems a priori to be casting doubts on our ability to detect Earth analogues in the habitable zone. However, using parameters of the crosscorrelation function it has been possible to correct the magnetic cycle effects to less than $1 \mathrm{~m} / \mathrm{s}$. In addition, for some domain of stellar masses (K-dwarfs), we observe that the amplitude of the radial velocity effect is vanishing despite quite noticeable magnetic cycles. Finally, we notice that the periods of magnetic cycles are much longer (about a factor 10) than the expected periods of habitable planets orbiting $\mathrm{K}$ dwarfs. We are thus still convinced that Doppler spectroscopy has the potential to detect rocky planets in the habitable zone of $\mathrm{K}$ dwarfs. 


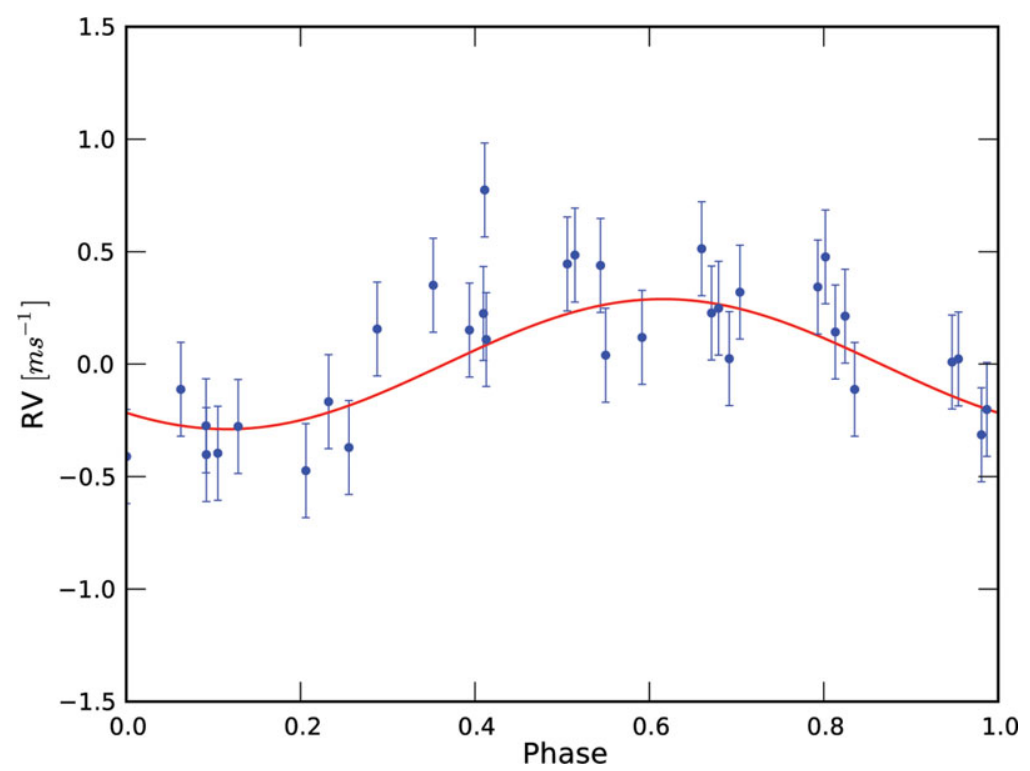

Figure 8. Simulation for the detection of a super-Earth of $2.5 M_{\oplus}$ in the habitable zone of an inactive K-dwarf (from Dumusque et al. 2011b).

The medium- or long-term scientific goal to search for chemical signatures of life in the atmospheric spectra of Earth twins via space experiments as the ESA-DARWIN concept will first require identification of targets. It seems that at the moment Doppler spectroscopy is the only method with the potential to detect Earth-type planets in the habitable zone of stars as close as possible to the Sun. The last condition is mandatory, if we want to have a star-planet angular separation large enough for the need of planetary atmosphere spectroscopy, as well as bright enough targets to maximize the signal-to-noise ratio.

From Doppler surveys we know that super-Earths on tight orbits are frequent. We have first hints from microlensing searches that super-Earths could also be frequent at large semi-major axis (Gould et al. 2010). But we do not have any estimate of the frequency of Earth-twins in the habitable zone of solar-type stars and no ideas on their orbital eccentricity distribution. The orbital eccentricity of Earth-twins is also relevant in the frame of life-search experiments. The ESA-PLATO space project is, in that context, the most interesting experiment, complementary to Doppler surveys to explore the domain of Earth-type planets orbiting relatively close stars.

\section{Acknowledgements}

We would like to thank the Swiss National Science Foundation for its continuous support.

\section{References}

Andrae, R., Schulze-Hartung, T., \& Melchior, P. 2010, arXiv:1012.3754v1

Correia, A., Udry, S., Mayor, M., et al. 2009, A\& $A$, 496, 521

Correia, A. C. M.., Couetdic, J., Laskar, J., et al. 2010, A\& A, 511, A21

Dumusque, X., Udry, S., Lovis, C., Santos, N. C., \& Monteiro, M. J. P.. F. G.. 2011a, A\&AA, 525, A140 
Dumusque, X., Santos, N. C., Udry, S., Lovis, C., \& Bonfils, X. 2011b, A\&A, 527, A82

Fischer, D. \& Valenti, J. 2005, ApJ, 622, 1102

Gould, A., Dong, S., Gaudi, B. S., et al. 2010, ApJ, 720, 1073

Gregory, P. C. 2011, MNRAS in press (arXiv:1101.0800)

Latham, D. W., Stefanik, R. P., Mazeh, T., Mayor, M., \& Burki, G. 1989, Nature, 339, 38

Lovis, C., Mayor, M., Pepe, F., et al. 2006, Nature, 441, 305

Lovis, C. \& Pepe, F. 2007, A\&3A, 468, 1115

Lovis, C., Mayor, M., Bouchy, F., Pepe, F., Queloz, D., Udry, S., Benz, W., \& Mordasini, C. 2009, Proc. IAU Symp., 253, 502

Lovis, C., Ségransan, D., Mayor, M., et al. 2011, A\&A, 528, A112

Mayor, M., Pepe, F., Queloz, D., et al. 2003, The Messenger, 114, 20

Mayor, M. \& Queloz, D. 1995, Nature, 378, 355

Mayor, M. \& Udry, S. 2008, Physica Scripta, 130, 014010

Mayor, M., Bonfils, X., Forveille., T., et al. 2009a, A\&SA, 507, 487

Mayor, M., Udry, S., Lovis, C., et al. 2009b, A\&A, 493, 639

Mordasini, C., Alibert, Y., \& Benz, W. 2009a, A\&AA, 501, 1139

Mordasini, C., Alibert, Y., Benz, W., \& Naef, D. 2009b, A\&A, 501, 1161

Pasquini, L., Cristiani, S., Garcia-Lopez, R., Haehnelt, M., \& Mayor, M. 2010, The Messenger, 140,20

Pepe, F., Correia, A. C. M., Mayor, M., et al. 2007, A\& $A, 462,769$

Pepe, F., Cristiani, S., Rebolo Lopez, R., et al. 2010, Proc. SPIE, 7735, 14

Pepe, F., et al. 2011, A\&A, submitted

Rivera, E. J., Lissauer, J. J., Butler, R. P., et al. 2005, ApJ, 634, 625

Santos, N. C., Israelian, G., \& Mayor, M. 2001, A\&A, 373, 1019

Santos, N. C., Israelian, G., \& Mayor, M. 2004, $A \mathscr{E} A, 415,1153$

Santos, N. C., Bouchy, F., Mayor, M., et al. 2004, A\& $A$, 426, L19

Sousa, S. G., Santos, N. C., Mayor, M., et al. 2008, A\&A A, 487, 373

Udry, S., Mayor, M., Benz, W., et al. 2006, A\&A A, 447, 361

Udry, S., Bonfils, X., Delfosse, X., et al. 2007, A\& $A$, 469, L43

Udry, S. \& Mayor, M. 2008, ASP Conf. Ser., 398, 13

Vogt, S. S., Butler, R. P., Rivera, E. J., Haghighipour, N. H., Gregory, W. W., \& Michael, H. 2010, ApJ, 723, 954

Wilken, T., Lovis, C., Manescau, A., et al. 2010, MNRAS, 405, L16 